\title{
Resource Allocation in MIMO-OFDMA Wireless Systems Based on Linearly Precoded Orthogonal Space-Time Block Codes
}

\author{
Borja Dañobeitia, Guillem Femenias, and Felip Riera-Palou* \\ Mobile Communications Group, University of the Balearic Islands \\ Ctra. de Valldemossa, km. 7,5, 07122 Palma (Illes Balears), Spain \\ \{borja.danobeitia, guillem.femenias, felip.riera\}@uib.es
}

\begin{abstract}
This paper proposes and analyzes efficient rate and power allocation algorithms for MIMO-OFDMA broadcast channels based on linearly precoded orthogonal space-time block codes. The weighted sumrate maximization problem is considered for both continuous and discrete rate allocation schemes. Since this problem is non-convex and combinatorial in nature, the Lagrange dual decomposition method is used to find an accurate near-optimal solution. The effects of using different MIMO configurations and realistic channel models with space and frequency correlation over the weighted sum-rate of the system is considered.
\end{abstract}

Keywords: Resource allocation, OFDMA, MIMO, STBC, linear precoding, Lagrange dual methods.

\section{Introduction}

The use of multiple-input multiple-output (MIMO) technology has shown to be a major breakthrough in providing high-rate reliable wireless communications links [1,2, 3. Furthermore, orthogonal frequency division multiple access (OFDMA), which decouples a broadband frequency-selective channel into multiple parallel frequency-flat fading channels through the use of the fast Fourier transform (FFT), has demonstrated to provide a high degree of flexibility in radio resource allocation, allowing the exploitation of the so-called multiuser diversity embedded in frequency-selective fading channels 4 .

Over the past decade, owing to their great promises, OFDMA and MIMO have been synergistically integrated to offer the benefits of both resource allocation flexibility and high performance [5, 6] and MIMO-OFDMA systems have found their way into several standards for next-generation wireless communication networks, notably IEEE 802.16e and 3GPP-LTE (Third Generation Partnership Project-Long Term Evolution). There are different ways of exploiting

\footnotetext{
* This work has been supported in part by the Ministerio de Ciencia e Innovación, Spain, and FEDER, under grant TEC2008-02422 and a PhD grant from Conselleria d'Economia, Hisenda i Innovació del Govern de les Illes Balears.
} 
multiple antennas at both ends of the MIMO-OFDMA communications link; among them, orthogonal space-time block coding (OSTBC) [7 is a simple yet very effective means of achieving transmit/receive diversity. These space-time coding schemes do not exploit channel knowledge at the transmitter. However, channel state information (CSI), if it is available, can of course be used to combine linear precoding strategies with OSTBC, exploiting in this way the joint benefits of both conventional beamforming and OSTBC [8, 9, 10].

Based on the analytical framework proposed by Seong et al. [11, our objective in this paper is to propose and analyze efficient rate and power allocation algorithms for MIMO-OFDMA broadcast channels based on the use of combined linear precoding and OSTBC. In particular, the weighted sum-rate maximization problem is considered. It is a non-convex combinatorial problem whose complexity increases exponentially with the number of users and subcarriers in the system [12. However, Yu and Lui [13] showed that, even though this optimization problem is non-convex, the Lagrange dual decomposition method can be used to find an accurate suboptimal solution, as the duality gap becomes zero when the number of subcarriers goes to infinity. Motivated by this result, the downlink weighted sum-rate maximization problem for linearly precoded OSTBC-based MIMO-OFDMA wireless systems is solved in the dual domain by using Lagrange dual decomposition.

\section{System Model}

Let us consider the downlink of a single-cell1 OFDMA base station equipped with $N_{T}$ transmit antennas. We note that these $N_{T}$ physical antennae will be transformed into $M$ (with $M \leq N_{T}$ ) virtual antennas through the action of the precoder. The OFDMA system has $N_{\text {FFT }}$ subcarriers from which $N$ of them are used to provide service to $K$ active mobile stations, each equipped with $N_{R}$ receive antennas. Used subcarriers and active users are indexed by the sets $\mathcal{N}=\{0, \ldots, N-1\}$ and $\mathcal{K}=\{0, \ldots, K-1\}$, respectively. Let us also assume that user $k$ has been assigned subcarrier $n$, and that the base station uses an OSTBC in order to achieve transmit diversity. We denote the code matrix that defines the OSTBC as $\boldsymbol{C}\left(\boldsymbol{Z}_{k, n, \eta}\right) \in \mathbb{C}^{T \times M}$, which is used to distribute the $\eta$ th block of $K_{s}$ symbols $\boldsymbol{Z}_{k, n, \eta}=\left\{z_{k, n, \eta, 0}, \ldots, z_{k, n, \eta, K_{s}-1}\right\}$ among the $M$ virtual transmit antennas and T OFDM symbol periods where, without loss of generality, symbols $\left\{z_{k, n, \eta, k_{s}}\right\}_{k_{s}=0}^{K_{s}-1}$ are assumed to be taken from a complex constellation $\mathcal{A}$ with average energy $\mathbb{E}\left\{\left|z_{k, n, \eta, k_{s}}\right|^{2}\right\}=1$. Since $T$ OFDM symbols are necessary to transmit $K_{s} \leq T$ symbols, the coding rate of the $\mathrm{STBC}$ is $R_{c}=K_{s} / T$. The elements of $\boldsymbol{C}\left(\boldsymbol{Z}_{k, n, \eta}\right)$ are linear combinations of the symbols $\left\{z_{k, n, \eta, k_{s}}\right\}_{k_{s}=0}^{K_{s}-1}$ and their conjugates. Furthermore, due to orthogonality, it holds that

$$
\left(\boldsymbol{C}\left(\boldsymbol{Z}_{k, n, \eta}\right)\right)^{H} \boldsymbol{C}\left(\boldsymbol{Z}_{k, n, \eta}\right)=a\left(\sum_{k_{s}=0}^{K_{s}-1}\left|z_{k, n, \eta, k_{s}}\right|^{2}\right) \boldsymbol{I}_{M},
$$

\footnotetext{
${ }^{1}$ In order to simplify the problem of resource allocation, the inter-cell interference is assumed to be either absent or simply modeled as additive white Gaussian noise.
} 
where $(\cdot)^{H}$ denotes the matrix complex conjugate transpose operator, $\boldsymbol{I}_{M}$ is the $M \times M$ identity matrix and $a$ is a constant that depends on the OSTBC coding matrix. For instance, $a=1$ for the Alamouti-STBC and, taking the codes proposed by Tarokh et al. in [7, $a=1$ for the rate $R_{c}=3 / 4$ OSTBCs $\mathcal{H}_{3}$ and $\mathcal{H}_{4}$, and $a=2$ the rate $R_{c}=1 / 2$ OSTBCs $\mathcal{G}_{3}$ and $\mathcal{G}_{4}$. Before transmission, the space-time codeword is multiplied by a precoding matrix $\boldsymbol{F}_{k, n, \eta} \in \mathbb{C}^{M \times N_{T}}$, satisfying the power constraint $\left\|\boldsymbol{F}_{k, n, \eta}^{H} \boldsymbol{F}_{k, n, \eta}\right\|_{F}^{2}=1$, where $\|\cdot\|_{F}$ denotes the matrix Frobenius norm.

At the receiver side, assuming perfect sample and symbol synchronization, and a cyclic prefix of length greater that the maximum duration of the channel impulse response, the $T \times N_{R}$ received signal matrix for the OSTBC block $\eta$ of user $k$ over subcarrier $n$ is given by

$$
\boldsymbol{Y}_{k, n, \eta}=\sqrt{\frac{p_{k, n, \eta}}{a R_{c}}} \boldsymbol{C}\left(\boldsymbol{Z}_{k, n, \eta}\right) \boldsymbol{F}_{k, n, \eta} \boldsymbol{H}_{k, n, \eta}+\boldsymbol{\nu}_{k, n, \eta},
$$

where $p_{k, n, \eta}$ is the power allocated to user $k$ over subcarrier $n$ for each OFDM symbol in OSTBC block period $\eta$ and the receiver noise $\boldsymbol{\nu}_{k, n, \eta}$ is an $T \times N_{R}$ matrix with elements modeled as independent identically distributed (i.i.d.) zeromean complex circular-symmetric Gaussian random variables, each with variance $\sigma_{\nu}^{2}$. The matrix $\boldsymbol{H}_{k, n, \eta}$ denotes the $N_{T} \times N_{R}$ frequency domain complex channel gain matrix from all transmit antennas to all receive antennas for user $k$ over subcarrier $n$ and OSTBC block period $\eta$. The elements of $\boldsymbol{H}_{k, n, \eta}$ are derived from a user-dependent power delay profile [14,15]. Assuming perfect CSI, the maximum likelihood (ML) receiver decides in favor of the block of symbols $\hat{\boldsymbol{Z}}_{k, n, \eta}$ satisfying

$$
\hat{\boldsymbol{Z}}_{k, n, \eta}=\arg \min _{\hat{\boldsymbol{Z}} \in \mathcal{A}^{K_{s}}}\left\|\boldsymbol{Y}_{k, n, \eta}-\sqrt{\frac{p_{k, n, \eta}}{a R_{c}}} \boldsymbol{C}(\hat{\boldsymbol{Z}}) \boldsymbol{F}_{k, n, \eta} \boldsymbol{H}_{k, n, \eta}\right\|^{2} .
$$

Noting the column orthogonal characteristic of the space-time block coding matrix, the minimization in (3) can be decoupled into $K_{s}$ parts that are only a function of $z_{k, n, \eta, k_{s}}$, for $k_{s}=0, \ldots, K_{s}-1$, respectively [7,16]. It can be shown that the ML decision rule for the $k_{s}$ th symbol $z_{k, n, \eta, k_{s}}$ is given by [16]

$$
\hat{z}_{k, n, \eta, k_{s}}=\arg \min _{\hat{z} \in \mathcal{A}}\left|\tilde{y}_{k, n, \eta, k_{s}}-\sqrt{\frac{p_{k, n, \eta}}{R_{c}}}\left\|\boldsymbol{F}_{k, n, \eta} \boldsymbol{H}_{k, n, \eta}\right\|_{F}^{2} \hat{z}\right|^{2},
$$

for all $k_{s}=0, \ldots, K_{s}-1$, with

$$
\tilde{y}_{k, n, \eta, k_{s}}=\sqrt{\frac{p_{k, n, \eta}}{R_{c}}}\left\|\boldsymbol{F}_{k, n, \eta} \boldsymbol{H}_{k, n, \eta}\right\|_{F}^{2} z_{k, n, \eta, k_{s}}+\tilde{\nu}_{k, n, \eta, k_{s}},
$$

where $\tilde{\nu}_{k, n, \eta, k_{s}}$ is a noise sample modeled as a zero-mean complex circularsymmetric Gaussian random variable with variance $\sigma_{\nu}^{2} \alpha_{k, n, \eta}$. Thus, the effective instantaneous signal to noise ratio at the output of the ML detector can be expressed as

$$
\gamma_{k, n, \eta}=\frac{p_{k, n, \eta} \alpha_{k, n, \eta}}{R_{c} \sigma_{\nu}^{2}}
$$


where $\alpha_{k, n, \eta}=\left\|\boldsymbol{F}_{k, n, \eta} \boldsymbol{H}_{k, n, \eta}\right\|_{F}^{2}$. The optimal precoding matrix, in the sense of maximizing the SNR, is thus obtained when $\alpha_{k, n, \eta}$ is maximized. As stated by Sanayei et al. in [10, Theorem 1], an optimal precoding matrix has the form 2 $\boldsymbol{F}_{k, n, \eta}=\boldsymbol{\psi} \boldsymbol{v}_{k, n, \eta}^{T}$, where $\boldsymbol{v} \in \mathbb{C}^{N_{T} \times 1}$ is the left singular vector of $\boldsymbol{H}_{k, n, \eta}$ associated with its largest singular value, denoted as $\sigma_{\max }\left(\boldsymbol{H}_{k, n, \eta}\right)$, and $\boldsymbol{\psi} \in \mathbb{C}^{M \times 1}$ is an arbitrary unit norm vector. For the above choice of $\boldsymbol{F}_{k, n, \eta}$ we have that

$$
\begin{aligned}
\alpha_{k, n, \eta} & =\operatorname{trace}\left(\boldsymbol{H}_{k, n, \eta}^{H} \boldsymbol{F}_{k, n, \eta}^{H} \boldsymbol{F}_{k, n, \eta} \boldsymbol{H}_{k, n, \eta}\right) \\
& =\operatorname{trace}\left(\boldsymbol{H}_{k, n, \eta}^{H} \boldsymbol{v}_{k, n, \eta}\|\boldsymbol{\psi}\|_{2}^{2} \boldsymbol{v}_{k, n, \eta}^{T} \boldsymbol{H}_{k, n, \eta}\right)=\sigma_{\max }^{2}\left(\boldsymbol{H}_{k, n, \eta}\right) .
\end{aligned}
$$

\section{Optimization Variables}

Power allocation: Let $\boldsymbol{p}_{n, \eta}=\left[p_{0, n, \eta} \cdots p_{K-1, n, \eta}\right]^{T}$ denote the vector of power allocation values for subcarrier $n$ and STBC block $\eta$. For a given set of constraints, the allocation algorithm will be in charge of determining the power allocation vector

$$
\boldsymbol{p}_{\eta}=\left[\left(\boldsymbol{p}_{0, \eta}\right)^{T} \cdots\left(\boldsymbol{p}_{N-1, \eta}\right)^{T}\right]^{T}
$$

optimizing the objective function. In addition to determining the power allocation values, the resource allocation algorithms should also allocate subcarriers and transmission rates. Nevertheless, as it will be shown next, the power allocation vector $\boldsymbol{p}_{\eta}$ can also be used to represent the allocation of all these resources, thus simplifying the formulation of the optimization problem [17].

Subcarrier allocation: As usual, it is assumed that subcarrier sharing is exclusive, that is, only one user is allowed to transmit on a given subcarrier. Hence, the subcarrier allocation constraints can be captured by constraining the power allocation vector as

$$
\boldsymbol{p}_{n, \eta} \in \mathcal{P}_{n}=\left\{\boldsymbol{p}_{n, \eta} \in \mathbb{R}_{+}^{K}: p_{k, n, \eta} p_{k^{\prime}, n, \eta}=0 ; \forall k \neq k^{\prime} ; k, k^{\prime} \in \mathcal{K}\right\} .
$$

Hence, the power allocation vector satisfies

$$
\boldsymbol{p}_{\eta} \in \mathcal{P}=\mathcal{P}_{0} \times \cdots \times \mathcal{P}_{N-1} \subset \mathbb{R}_{+}^{K N} .
$$

Continuous rate allocation: The achievable capacity (ideal continuous rate) for user $k$ over subcarrier $n$, measured in bits per second per $\mathrm{Hz}$, can be written as

$$
\begin{aligned}
R_{k, n, \eta}\left(p_{k, n, \eta}, \alpha_{k, n, \eta}\right) & =R_{c} \log _{2}\left(1+\gamma_{k, n, \eta}\right) \\
& =R_{c} \log _{2}\left(1+\frac{p_{k, n, \eta} \alpha_{k, n, \eta}}{R_{c} \sigma_{\nu}^{2}}\right) .
\end{aligned}
$$

As a consequence, the transmission rate allocation is uniquely determined by the power allocation value $p_{k, n, \eta}$.

\footnotetext{
${ }^{2}$ Notice that we have defined the channel matrix as the transpose of that used in 10 . Furthermore, the objective of minimizing the error probability, as used in [10], is equivalent to our objective of maximizing the SNR.
} 
Discrete rate allocation: Systems based on adaptive modulation and coding (AMC) can only use a discrete set $\mathcal{M}=\{0, \ldots, M-1\}$ of modulation and coding schemes (MCS), which are characterized by a particular transmission rate $r_{m}$ (in bits per second per Hertz), for all $m \in \mathcal{M}$. In this case, the set $\mathbb{R}^{+}$is subdivided in $M$ disjoint intervals, each defining the margin of effective instantaneous signal to noise ratios over which a particular MCS will be selected by the AMC scheme. Thus, the transmission rate for user $k$ over subcarrier $n$ and STBC block $\eta$ can be expressed using the staircase function

$$
R_{k, n, \eta}\left(p_{k, n, \eta}, \alpha_{k, n, \eta}\right)=\left\{\begin{array}{cc}
R_{c} r_{0} & , 0 \leq \gamma_{k, n, \eta}<\Gamma_{1} \\
R_{c} r_{1} & , \Gamma_{1} \leq \gamma_{k, n, \eta}<\Gamma_{2} \\
\vdots & \vdots \\
R_{c} r_{M-1} & , \Gamma_{M-1} \leq \gamma_{k, n, \eta}<\infty
\end{array}\right.
$$

where $\left\{\Gamma_{m}\right\}_{m=1}^{M-1}$, with $\Gamma_{m+1} \geq \Gamma_{m}$, are the effective instantaneous SNR boundaries defining the MCS selection intervals. Generally, these SNR boundaries are obtained by setting a target error rate $P_{0}$ and determining the margin of effective instantaneous SNR values that guarantee the target error rate when using each one of the MCSs. As in the continuous rate case, the power allocation value $p_{k, n, \eta}$ uniquely determines the transmission rate allocation.

\section{Weighted Sum-Rate Maximization}

Assuming the availability of perfect channel state information (CSI), the instantaneous weighted sum-rate maximization problem can be formulated as (see, for instance, [18, 11, 17])

$$
\begin{array}{ll}
\max _{\boldsymbol{p}_{\eta} \in \mathcal{P}} & \sum_{k=0}^{K-1} w_{k, \eta} \sum_{n=0}^{N-1} R_{k, n, \eta}\left(p_{k, n, \eta}, \alpha_{k, n, \eta}\right) \\
\text { s.t. } & \sum_{k=0}^{K-1} \sum_{n=0}^{N-1} p_{k, n, \eta} \leq P_{T},
\end{array}
$$

where $P_{T}$ is the maximum base station transmission power. The user weights $\left\{w_{k, \eta}\right\}_{k=0}^{K-1}$ are positive constants satisfying $\sum_{k=0}^{K-1} w_{k, \eta}=1$ that allow the MAC layer setting priorities of different users in the system and enforcing certain notions of fairness [17]. Let us approach this optimization problem using duality principles 13. The Lagrangian of (13) can be expressed as

$$
\mathcal{L}\left(\boldsymbol{p}_{\eta}, \lambda\right)=\sum_{k=0}^{K-1} w_{k, \eta} \sum_{n=0}^{N-1} R_{k, n, \eta}\left(p_{k, n, \eta}, \alpha_{k, n, \eta}\right)+\lambda\left(P_{T}-\sum_{k=0}^{K-1} \sum_{n=0}^{N-1} p_{k, n, \eta}\right) .
$$

Using the subcarrier exclusive allocation constraint and the fact that the power variables are separable across subcarriers, the dual optimization problem can be 
written as

$$
\begin{aligned}
g(\lambda) & =\min _{\lambda \geq 0}\left[\max _{\boldsymbol{p}_{\eta} \in \mathcal{P}} \mathcal{L}\left(\boldsymbol{p}_{\eta}, \lambda\right)\right] \\
& =\min _{\lambda \geq 0}\left[\lambda P_{T}+\sum_{n=0}^{N-1} \max _{k \in \mathcal{K}}\left(\max _{p_{k, n, \eta} \geq 0}\left(w_{k, \eta} R_{k, n, \eta}\left(p_{k, n, \eta}, \alpha_{k, n, \eta}\right)-\lambda p_{k, n, \eta}\right)\right)\right] .
\end{aligned}
$$

Thus, the problem has been reduced to a per-subcarrier optimization, and since $N \gg K$, the computational complexity has been significantly reduced.

Continuous rate allocation: In case of using $R_{k, n, \eta}\left(p_{k, n, \eta}, \alpha_{k, n, \eta}\right)$ as defined in (11), the innermost maximization in (15) provides a multilevel water-filling closed-form expression for the optimal power allocation given as

$$
\wp_{k, n, \eta}(\lambda)=\left[\frac{1}{\chi_{k, n, \eta}(\lambda)}-\frac{1}{\zeta_{k, n, \eta}}\right]^{+}
$$

where $[x]^{+}=\max (0, x), \chi_{k, n, \eta}(\lambda)=\frac{\lambda \ln 2}{w_{k, n, \eta} R_{c}}$ and $\zeta_{k, n, \eta}=\frac{\alpha_{k, n, \eta}}{R_{c} \sigma_{\nu}^{2}}$. Now, using (16) in (15) yields

$$
g(\lambda)=\min _{\lambda \geq 0}\left[\lambda P_{T}+\sum_{n=0}^{N-1} \max _{k \in \mathcal{K}}\left(w_{k, \eta} R_{k, n, \eta}\left(\wp_{k, n, \eta}(\lambda), \alpha_{k, n, \eta}\right)-\lambda \wp_{k, n, \eta}(\lambda)\right)\right] .
$$

Using standard properties of dual optimization problems 13, it can be shown that the objective function for the dual problem is convex with respect to $\lambda$ and thus, line search methods like, for example, Golden-section or Fibonacci, can be used to determine $\lambda^{*}$. Once $\lambda^{*}$ has been found, it can be used in the optimization functions to obtain the following user and power allocation for each of the subcarriers in the system

$$
\begin{gathered}
k_{n}^{*}=\arg \max _{k \in \mathcal{K}}\left(w_{k, \eta} R_{k, n, \eta}\left(\wp_{k, n, \eta}\left(\lambda^{*}\right), \alpha_{k, n, \eta}\right)-\lambda^{*} \wp_{k, n, \eta}\left(\lambda^{*}\right)\right) \\
p_{k, n, \eta}=\left\{\begin{array}{cl}
\wp_{k, n, \eta}\left(\lambda^{*}\right), & k=k_{n}^{*} \\
0 \quad, & \text { otherwise. }
\end{array}\right.
\end{gathered}
$$

Discrete rate allocation: In this case $R_{k, n, \eta}\left(p_{k, n, \eta}, \alpha_{k, n, \eta}\right)$ is a non-derivable discontinuous function. However, using (12) the set $\mathbb{R}^{+}$can be subdivided into $M$ segments

$$
\mathcal{R}_{m}^{+}=\left[\frac{\Gamma_{m}}{\zeta_{k, n, \eta}}, \frac{\Gamma_{m+1}}{\zeta_{k, n, \eta}}\right), m \in \mathcal{M}
$$

and given that $\lambda$ and $p_{k, n, \eta}$ belong to $\mathbb{R}^{+}$, if a power allocation $p_{k, n, \eta}$ is used such that $\Gamma_{m} \leq \gamma_{k, n, \eta}<\Gamma_{m+1}$ then

$$
\begin{aligned}
& w_{k, \eta} R_{k, n, \eta}\left(p_{k, n, \eta}, \alpha_{k, n, \eta}\right)-\lambda p_{k, n, \eta} \\
& \quad=w_{k, \eta} R_{c} r_{m}-\lambda p_{k, n, \eta} \leq w_{k, \eta} R_{c} r_{m}-\lambda \frac{\Gamma_{m}}{\zeta_{k, n, \eta}}, \forall p_{k, n, \eta} \in \mathcal{R}_{m}^{+} .
\end{aligned}
$$


As a consequence, there only exist $M$ candidate power allocations

$$
\wp_{k, n, \eta}(\lambda) \in\left\{\frac{\Gamma_{0}}{\zeta_{k, n, \eta}}, \ldots, \frac{\Gamma_{M-1}}{\zeta_{k, n, \eta}}\right\}
$$

from which the one maximizing $w_{k, \eta} R_{c} r_{m}-\lambda \frac{\Gamma_{m}}{\zeta_{k, n, \eta}}$ must be selected, that is,

$$
\wp_{k, n, \eta}(\lambda)=\frac{\Gamma_{m_{k, n}^{*}(\lambda)}}{\zeta_{n, k, \eta}}
$$

where

$$
m_{k, n}^{*}(\lambda)=\arg \max _{m \in \mathcal{M}}\left(w_{k, \eta} R_{c} r_{m}-\lambda \frac{\Gamma_{m}}{\zeta_{k, n, \eta}}\right) .
$$

Using (22) in (15), the dual optimization problem can be rewritten as

$$
g(\lambda)=\min _{\lambda \geq 0}\left[\lambda P_{T}+\sum_{n=0}^{N-1} \max _{k \in \mathcal{K}}\left(w_{k, \eta} R_{k, n, \eta}\left(\frac{\Gamma_{m_{k, n}^{*}(\lambda)}}{\zeta_{n, k, \eta}}, \alpha_{k, n, \eta}\right)-\lambda \frac{\Gamma_{m_{k, n}^{*}(\lambda)}}{\zeta_{n, k, \eta}}\right)\right]
$$

and, as in the continuous rate allocation case, simple line search methods can be used to determine $\lambda^{*}$, that can then be substituted in the optimization functions to obtain the optimal user, rate and power allocation for each of the subcarriers of the system

$$
\begin{array}{r}
k_{n}^{*}=\arg \max _{k \in \mathcal{K}}\left(w_{k, \eta} R_{c} r_{m_{k, n}^{*}\left(\lambda^{*}\right)}-\lambda^{*} \frac{\Gamma_{m_{k, n}^{*}\left(\lambda^{*}\right)}}{\zeta_{k, n, \eta}}\right) \\
R_{k, n, \eta}=\left\{\begin{array}{cl}
R_{c} r_{m_{k, n}^{*}\left(\lambda^{*}\right)}, & k=k_{n}^{*} \\
0 \quad, & \text { otherwise, }
\end{array} \quad p_{k, n, \eta}=\left\{\begin{array}{cl}
\frac{\Gamma_{m_{k, n}^{*}\left(\lambda^{*}\right)}}{\zeta_{k, n, \eta}}, & k=k_{n}^{*} \\
0 & \text { otherwise. }
\end{array}\right.\right.
\end{array}
$$

\section{Numerical Results}

Without loss of generality, we consider a MIMO-OFDMA system with parameters extracted from the draft specifications of IEEE 802.11n. In particular, we consider an operating setup with bandwidth $B=20 \mathrm{MHz}$ over a carrier frequency $f_{0}=5.25 \mathrm{GHz}$ and $N_{\mathrm{FFT}}=64$ subcarriers from which $N=52$ are used to transmit data. For simplicity, an uncoded Gray-mapped square $2^{r_{l}}$-QAM with $r_{l} \in\{0,2,4,6\}$ bits and SNR thresholds $\eta_{l} \in\{-\infty, 9.97,16.96,23.19\} \mathrm{dB}$ has been assumed in discrete rate allocation case [17. The use of channel coding certainly would increase the global sum-rate but would not modify the general trends and conclusions. The frequency-selective Rayleigh fading channel has been simulated using models B and E proposed by Kermoal et al. in [15. Transmit and receive antennas are configured as linear uniform arrays with antenna spacing of 1 and 1/2 wavelength, respectively. Numerical results have been obtained through a Monte-Carlo simulation using 1,000 channel realizations. 


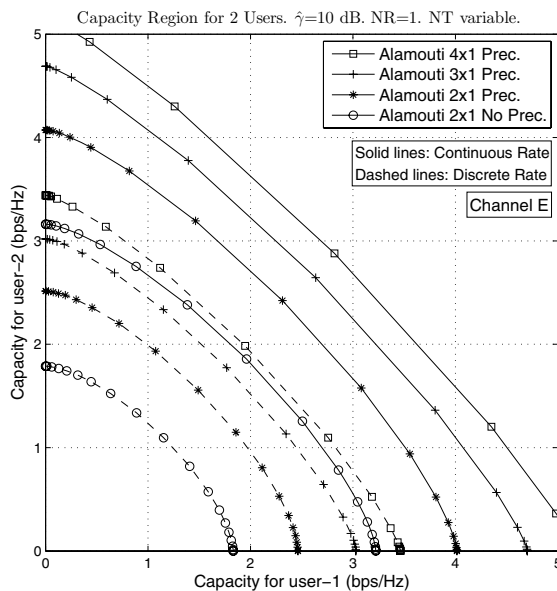

(a)

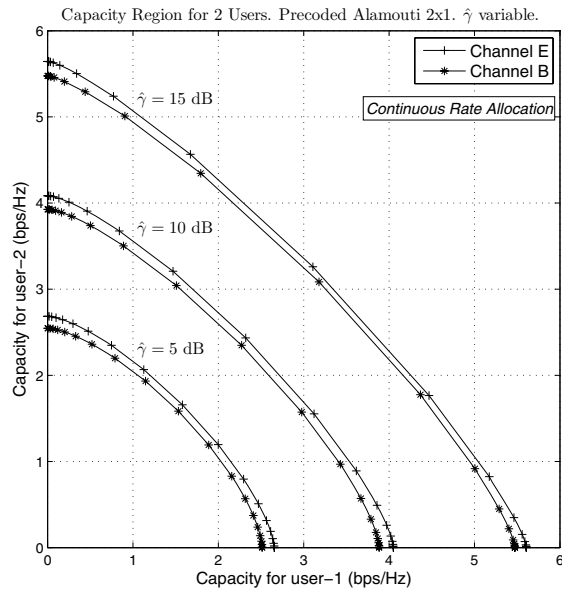

(c)

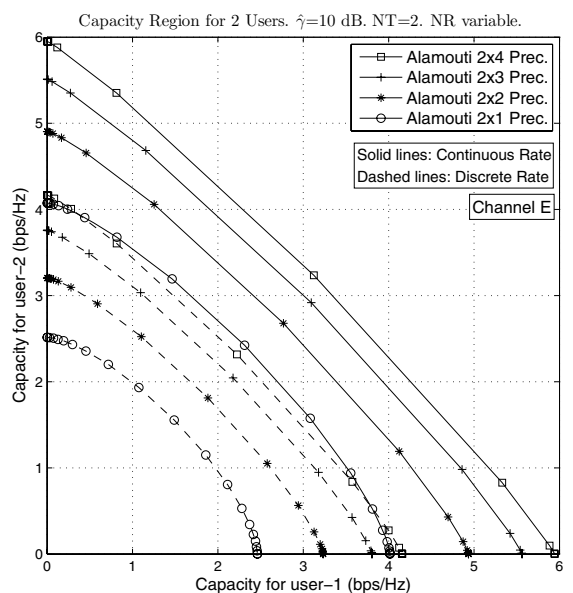

(b)

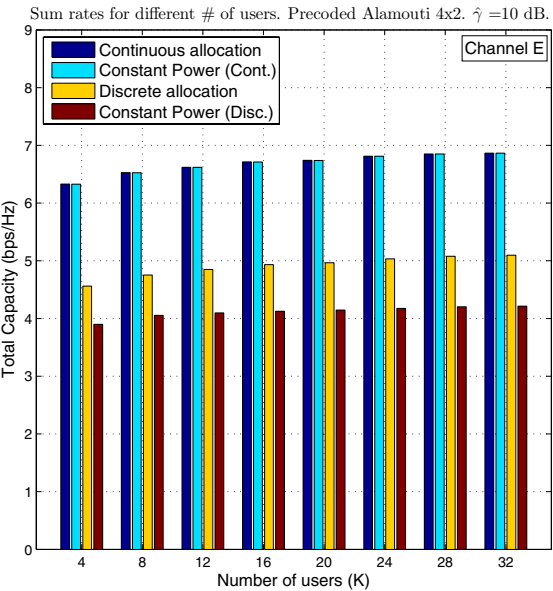

(d)

Fig. 1. Two-user capacity regions and sum rates for different number of users as a function of $N_{T} \times N_{R}$ MIMO configuration, frequency-selective channel model and resource allocation strategy

In Figs. 1(a) and 1(b), we show the capacity regions of a linearly precoded system using Alamouti STBC with 2 users and varying $w_{1}$ between 0 and 1 , and setting $w_{2}=1-w_{1}$. Results are presented for continuous and discrete rate allocation algorithms as a function of $N_{T} \times N_{R}$ MIMO configuration. Obviously, discrete rate allocation algorithms show a capacity loss with respect to the ideal (Shannon capacity-based) continuous rate allocation schemes. Nevertheless, as observed by Wong et al. in [17, the general trends are very similar to the continuous rate case. As shown in Fig. 1(a), the joint use of OSTBC and linear 
precoding at the transmitter side produces a substantial increase in system capacity. Fig. 1(b) shows an even more pronounced capacity increase as the number of receive antennas is incremented. However, it is important to note the diminishing capacity returns that are obtained as the number of diversity branches increases, either at the transmitter or the receiver sides. Fig. 1(c) shows the same capacity regions for different values of $\hat{\gamma} \triangleq P_{T} / \sigma_{\nu}^{2}$ and varying the frequencyselective Rayleigh channel model. It can be observed that, especially for lower SNRs, a higher sum rate is obtained when transmitting over channel model E. This suggests that channel selectivity (spatial and/or frequential) can be converted into multiuser diversity.

Fig. 1(d) depicts the sum capacity for a precoded Alamouti $4 \times 2$ system, with $\hat{\gamma}=10 \mathrm{~dB}$, as a function of the number of users, $K$. Results have been obtained by generating a random set of user weights for each channel realization. Sum capacity results for a constant power allocation strategy are also shown for comparison. First of all, the effect of multiuser diversity can be observed as the sum capacity grows as the number of users increases. Second, we see that the capacity gain due to multiuser diversity is also subject to the diminishing capacity returns as the number of users increases. Finally, it is interesting to note that the capacity gain provided by the use of variable power allocation algorithms becomes negligible when using continuous rate allocation algorithms, thus suggesting that using powerful channel coding strategies in a more realistic discrete rate system can make unnecessary the use of power allocation.

\section{Conclusions}

In this paper we have proposed and analyzed effective rate and power allocation strategies for MIMO-OFDMA broadcast channels in systems employing linearly precoded orthogonal space-time block codes. The Lagrange dual decomposition method has been used to find an accurate near-optimal solution to the weighted sum-rate maximization problem for both continuous and discrete rate allocation schemes. Simulations have been presented for different MIMO configurations, realistic frequency-selective channel profiles and varying number of users. Results demonstrate that the joint use of OSTBC and linear precoding at the transmitter side and space diversity at receiver side produces a substantial increase in system capacity. However, diminishing capacity returns are obtained as the number of diversity branches increases, either at transmission or reception. Similarly, multiuser diversity is also affected by diminished capacity returns as the number of users grows.

\section{References}

1. Gesbert, D., Shafi, M., Shiu, D., Smith, P.J., Naguib, A.: From theory to practice: an overview of MIMO space-time coded wireless systems. IEEE JSAC 21(3), 281-302 (2003) 
2. Goldsmith, A., Jafar, S.A., Jindal, N., Vishwanath, S.: Capacity limits of MIMO channels. IEEE JSAC 21(5), 684-702 (2003)

3. Paulraj, A.J., Gore, D.A., Nabar, R.U., Bolcskei, H.: An overview of MIMO communications-a key to gigabit wireless. Proc. IEEE 92(2), 198-218 (2004)

4. Wang, Z., Giannakis, G.B.: Wireless multicarrier communications. IEEE Signal Proc. Mag. 17(3), 29-48 (2000)

5. Stuber, G.L., Barry, J.R., Mclaughlin, S.W., Li, Y.G., Ingram, M.A., Pratt, T.G.: Broadband MIMO-OFDM wireless communications. Proc. IEEE 92(2), 271-294 (2004)

6. Bolcskei, H.: MIMO-OFDM wireless systems: basics, perspectives, and challenges. IEEE Wireless Communications 13(4), 31-37 (2006)

7. Tarokh, V., Jafarkhani, H., Calderbank, A.R.: Space-time block coding for wireless communications: performance results. IEEE JSAC 17(3), 451-460 (1999)

8. Skoglund, G.J.M., Ottersten, B.: Combining beamforming and orthogonal spacetime block coding. IEEE Tran. Inf. Theory. 48(3), 611-627 (2002)

9. Love, D.J., Heath, J.R.W.: Limited feedback unitary precoding for orthogonal space-time block codes. IEEE Tran. Sig. Proc. 53(1), 64-73 (2005)

10. Sanayei, S., Love, D.J., Nosratinia, A.: On the design of linear precoders for orthogonal space-time block codes with limited feedback. In: Proc. IEEE WCNC, pp. 489-493 (2005)

11. Seong, K., Mohseni, M., Cioffi, J.M.: Optimal resource allocation for OFDMA downlink systems. In: Proc. International Symposium on Information Theory (ISIT), July 2006, pp. 1394-1398 (2006)

12. Hoo, L.M.C., Halder, B., Tellado, J., Cioffi, J.M.: Multiuser transmit optimization for multicarrier broadcast channels: asymptotic FDMA capacity region and algorithms. IEEE Tran. Commun. 52(6), 922-930 (2004)

13. $\mathrm{Yu}, \mathrm{W}$., Lui, R.: Dual methods for nonconvex spectrum optimization of multicarrier systems. IEEE Tran. Commun. 54(7), 1310 (2006)

14. Proakis, J.G.: Digital Communications, 4th edn. McGraw-Hill, New York (2001)

15. Kermoal, J., Schumacher, L., Pedersen, K., Mogensen, P., Frederiksen, F.: A stochastic MIMO radio channel model with experimental validation. IEEE JSAC 20(6), 1211-1226 (2002)

16. Femenias, G.: BER performance of linear STBC from orthogonal designs over MIMO correlated Nakagami-m fading channels. IEEE Tran. Veh. Technol. 53(2), 307-317 (2004)

17. Wong, I., Evans, B.: Resource allocation in multiuser multicarrier wireless systems. Springer, Heidelberg (2008)

18. Shen, Z., Andrews, J.G., Evans, B.L.: Optimal power allocation in multiuser OFDM systems. In: Proc. IEEE Global Telecommunications Conference (GLOBECOM), December 2003, pp. 337-341 (2003) 